\title{
Computational Techniques for The Evaluation of Inhomogeneity Parameters on Transient Conduction in Functionally Graded Layers
}

\author{
Mehmet N. Balci ${ }^{*}$, Barışs Sabuncuoğlu \\ Mechanical Engineering Department, Hacettepe University, 06800, Ankara, Turkey \\ *E-mail address: mehmetbalci@hacettepe.edu.tr, barissabuncuoglu@hacettepe.edu.tr \\ ORCID numbers of authors: \\ 0000-0002-4416-6761*, 0000-0001-5156-746X
}

Received date: 17.09 .2019

Accepted date: 01.11.2019

\begin{abstract}
Transient thermal response of a functionally graded material (FGM) layer is considered and individual effects of inhomogeneity parameters on temperature distribution are examined. Transient conduction equation has variable coefficients controlling conductivity, mass density and specific heat capacitance due to the material property variation along the thickness of the graded layer. In order to solve the time dependent conduction equation for the unknown interior temperatures, computational methods are employed based on finite difference and finite element methods. Governing partial differential equation is discretized in space and time grids and computer codes are developed to implement explicit and implicit schemes. Results of explicit and implicit schemes are compared with those found by finite element method. A very good agreement is achieved for the applied boundary and initial conditions. Parametric study reveals the individual influences of various inhomogeneity parameters of FGM upon time dependent temperature distribution of a functionally graded layer. The results of the direct comparison study indicate that inhomogeneity parameters for specific heat and mass density have greater influence on temperature distribution than that for thermal conductivity.
\end{abstract}

Keywords: Functionally Graded Layer, Transient Heat Conduction, Computational Methods, Material Inhomogeneity

\section{Introduction}

Functionally graded materials (FGMs) are advanced composites involving two or more constituent phases. These materials are regarded as advanced engineering composites designed to meet material demands with the variance of via the spatial gradation in the structure. Originally considered as thermal barrier coatings for aerospace structures and fusion reactors, FGMs were also used as structural components in transportation, energy, electronics and biomedical engineering for the general use in high temperature environment in the last decade [1]. Generally, there are three different methods to obtain compositional gradient in the material which includes gas based, liquid phase and solid phase methods. Hence, physically or chemically tailored properties are obtained in the structure. Chemical Vapor Deposition (CVD), Physical Vapor Deposition (PVD), ion plating, plasma spraying and ion mixing are some examples of gas-based methods to fabricate FGMs. Methods such as centrifugal casting, slip casting, chemical solution deposition (CSD), electrochemical 
gradation are some liquid phase examples to product FGMs. Furthermore, spark plasma sintering and powder metallurgy techniques can be given for examples of solid phase methods [2]. Bellur-Ramaswamy et al. [3] developed an algorithm to optimize continuous quench process parameters to produce functionally graded aluminum alloy extrudes. The gradual changes in volume fraction of the constituents and non-homogenous structure provides continuous graded macroscopic properties, such as hardness, wear resistance, corrosion resistivity, thermal conductivity, specific heat and mass density that are critical for thermal barrier coatings (TBCs) as well as thermal protection of the re-entry capsule, furnace liners, body armour, piezoelectric actuators and electromagnetic sensors [4-7]. Therefore, there have been many studies related with the thermal properties of these materials and their interaction with their structural behavior. Numerical methods are frequently applied to investigate such properties to understand their thermal behavior in the design stage. It was reported that repeated hot gas flow tests indicated that utilization of FGM thermal barrier coatings (TBCs) enhanced the resistance to cracking and delamination at high temperatures when compared to conventional coatings. For example, $\mathrm{ZrO}_{2} / \mathrm{Ni}$ functionally graded material (FGM) was used as TBC for the rocket engine and no delamination was observed after 550 seconds of combustion. $\mathrm{ZrO}_{2}$ stabilized with $\mathrm{Y}_{2} \mathrm{O}_{3}$ functionally graded material (FGM) was used as TBC for turbine blades and it was seen that this material performed excellent resistance to erosion and thermal shock [8-9]. Reddy and Chin [10] investigated the dynamic thermoelastic response of functionally graded cylinders and plates, thermomechanical coupling was included and a finite element model was developed. Yang [11] proposed a research based on finite element analysis to examine the temperature distribution, thermal stresses and failure criteria of a multi-dimensional functionally graded material (FGM) plate which was composed of $\mathrm{ZrO}_{2}$ and Ti-6Al-4V and $\mathrm{Al}_{2} \mathrm{O}_{3}$ under steady-state, heating and sudden cooling conditions. Analytical method was proposed to analyze the transient heat conduction analysis in a cylindrical shell made of functionally graded material where material properties vary with the power law through the thickness [12]. The response of a circular cylindrical thin shell made of the functionally graded material (FGM) based on the generalized theory of thermoelasticity was determined. Power law was adopted for the spatial variation of the thermoelastic properties [13]. Mechanical and thermal buckling of FGM conical shell panels made of $\mathrm{Al} / \mathrm{ZrO}_{2}, \mathrm{SUS} 304 / \mathrm{Si}_{3} \mathrm{~N}_{4}$ and $\mathrm{Al}_{2} \mathrm{O}_{3} / \mathrm{Ti}-6 \mathrm{Al}-4 \mathrm{~V}$ were investigated through an elementfree method by Zhao and Liew [14].

Sharma et al. [15] constructed a finite element model to investigate the steady state temperature field in FGM layer which was composed of Zirconia and Aluminum. Through the thickness temperature distribution and thermal stresses in a plate which was made of functionally graded material were determined by Cho and Oden [16] using Crank-NicolsonGalerkin scheme. It was reported in the study conducted by Nemat-Alla [17] that twodimensional graded materials (2D-FGMs) had a great potential for minimization of temperatures, thermal and residual stresses under a severe thermal loading cycle that includes of heating followed by cooling operations. Sladdek et al. [18] proposed an advanced computational method based on local boundary integral equations for transient heat conduction analysis in continuously non-homogenous functionally graded materials (FGMs). Sadowski and Nakonieczny [19] focused on numerical study based on researching the FGM grading pattern impact on the temperature distribution in the cylindrical plates made of $\mathrm{Al}_{2} \mathrm{O}_{3} / \mathrm{ZrO}_{2}$ under the thermal shock condition. A meshfree, semi-discrete finite element method was proposed for the solution of the thermal shock problem for a thin, cylindrical plate made of FGM ceramics, and an explicit finite difference method was utilized for the temporal discretization [20]. A multiple reciprocity boundary face method was developed to investigate the transient heat conduction analysis of functionally graded materials [21]. Li and 
Wen [22] put forward a finite block method based on the Lagrange series to solve transient heat conduction problem in a functionally graded media regarding one dimensional to three dimensional conduction cases. A thermal conduction analysis of layered functionally graded materials composed of $\mathrm{Ni}$ and Carbon nanotube was performed by Olatunji- Ojo et al. [23] using a finite element code and parametric studies were carried out using different cooling times, different mixing rules and different heat transfer coefficients. Jin [24] examined the transient heat conduction in a functionally graded plate subjected to gradual cooling and heating at its boundaries and asymptotic analysis and integration technique were used to obtain a closed form asymptotic solution of temperature field in FGM plate for short times. Determination of through the thickness temperature distribution in functionally graded materials (FGMs) has significant importance since it directly influences the failure mechanism.

In the present study, we develop computer codes based on explicit and implicit schemes to determine the transient temperature distribution in a layer made of FGM and compare results with those obtained through the finite element analysis. Then, the separate influences of inhomogeneity parameters for the thermal conductivity, thermal capacitance and the mass density on temperature distribution in a graded layer are determined. The main novelty of the present study is the investigation of the influences of these inhomogeneity constants separately on the temperature distribution in FGM layers, individually and determination of the dominance of each parameter. Governing partial differential equation has variable coefficients of each material property and solution is performed keeping the generality. It is demonstrated that computational methods based on explicit and implicit schemes can efficiently be applied to such conduction problems including non-homogenous materials and they are able to provide fast and accurate solutions. In addition to that, although there have been many works in the literature which utilized some kind of different computational methods to examine the transient heat conduction problem in FGM layers, none of them compared the results of developed computational techniques with each other. Thus, another novelty of the paper is providing the comparison of the temperature results for an FGM layer obtained by explicit, implicit and FEA methods. It is believed that results of this study will be helpful for material designers to understand the transient thermal response of graded layers designated especially for thermal barrier coatings in harsh environments.

\section{FGM Material and Properties}

The functionally graded material (FGM) considered made of ceramic and metal phases is illustrated in Fig.1. One side of functionally graded layer is composed of $100 \%$ ceramic phase whereas the other side of the layer is composed of $100 \%$ metallic phase with the intermediate transition region in between. Material properties are denoted by exponential functions varying in $x$-axis. A schematic illustration of a continuously graded microstructure in FGM is provided by Chan et al. [25]. As provided in [26-27], thermal conductivity, heat capacitance and density of the functionally graded layer are defined by the exponential functions:

$$
\begin{aligned}
& k=k_{0} \exp \left(\gamma_{1} x\right), \\
& c=c_{0} \exp \left(\gamma_{2} x\right), \\
& \rho=\rho_{0} \exp \left(\gamma_{3} x\right) .
\end{aligned}
$$


where $k_{0}, c_{0}$ and $\rho_{0}$ are the thermal conductivity, heat capacitance and mass density at the metallic (left) surface $(x=0) \cdot \gamma_{1}, \gamma_{2}$ and $\gamma_{3}$ respectively show the inhomogeneity constants for thermal conductivity, specific heat capacitance and mass density and these constants are assumed to be different from each other in the present study. $\mathrm{ZrO}_{2}$ and Ti-6Al-4V are referencing ceramic and metallic materials utilized in the present study and their thermal properties are provided in Table 1.

Table 1. Material properties of $\mathrm{ZrO}_{2}$ and Ti-6Al-4V (Fujimoto and Noda [28]).

\begin{tabular}{|l|c|c|}
\hline Material & $\mathbf{Z r O}_{2}$ & Ti-6Al-4V \\
\hline Thermal conductivity $(\mathrm{W} / \mathrm{mK})$ & 2.036 & 18.1 \\
\hline Heat capacitance $(\mathrm{j} / \mathrm{kg} \mathrm{K})$ & 615.6 & 808.3 \\
\hline Density $\left(\mathrm{kg} / \mathrm{m}^{3}\right)$ & 5600 & 4420 \\
\hline
\end{tabular}

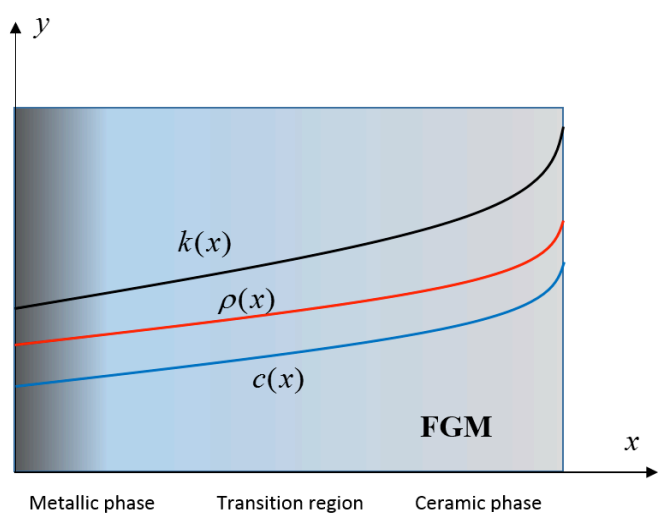

Fig. 1. The schematic illustration of functionally graded material possessing different inhomogeneity constants for thermal properties

\section{Equation of Conduction and Boundary Conditions}

In order to find the time dependent temperature distribution inside the FGM layer, the following heat conduction equation should be derived. One of the main contributions of this study over previous studies is to taking into consideration of spatial variation of each thermal properties separately, hence derived heat conduction equation is different that adopted in the previous studies. The derivation of conduction equation is shown in Eqs. (4)-(5) which involves variable parameters for thermal conductivity, heat capacitance and mass density.

$$
\begin{gathered}
\frac{\partial k(x)}{\partial x} \frac{\partial T}{\partial x}+k(x) \frac{\partial^{2} T}{\partial x^{2}}=\rho(x) c(x) \frac{\partial T}{\partial t} . \\
\frac{\partial T}{\partial t}=\gamma_{1} \alpha(x) \frac{\partial T}{\partial x}+\alpha(x) \frac{\partial^{2} T}{\partial x^{2}} .
\end{gathered}
$$

The thermal diffusivity for the FGM layer is expressed to be: 


$$
\begin{gathered}
\alpha(x)=\alpha_{0} \exp \left(\gamma_{1}-\gamma_{2}-\gamma_{3}\right) x \\
\alpha_{0}=\frac{k_{0}}{\rho_{0} c_{0}} .
\end{gathered}
$$

Two different boundary and initial condition cases are applied to the boundary value problem and these conditions are given in Table 2.

Table 2. Applied boundary and initial conditions

\begin{tabular}{|l|l|}
\hline Case 1 & Case 2 \\
\hline$T_{1}=0 K$, & $T_{1}=T_{0} K$, \\
\hline$T_{2}=0 K$, & $T_{2}=2 T_{0} K$, \\
\hline$T(x, 0)=T_{0} \times \sin (\pi x / L) K$ & $T(x, 0)=T_{0} K$ \\
\hline
\end{tabular}

where $T_{0}=1000 \mathrm{~K}$.

\section{Computational Techniques Applied for the Transient Thermal Analysis of FGM}

The addressed heat conduction equation is discretized utilizing the finite difference formula. Temperature at the left surface is denoted by $T_{0,0}$ and that at the right surface is shown by $T_{N, 0}$. This discretization in space and time parameters is illustrated in Fig. 2. After discretization step is completed, formulations for explicit and implicit schemes are developed.

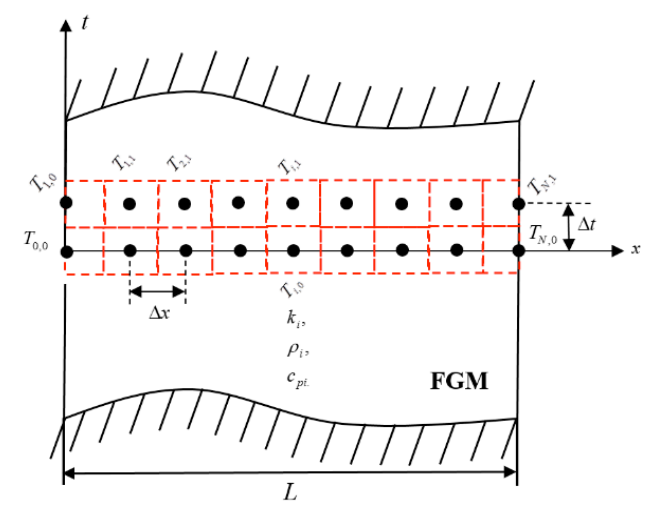

Fig. 2. Discretized numerical model prepared for the numerical analysis

In the following subsections 4.1 and 4.2, explicit and implicit formulations are provided. The derived formulations are implemented into MATLAB.

\subsection{Explicit method formulation}

The general explicit method finds the solution of node $i$ at time $j+1$ using the temperature information available at nodes $i-1, i$ and $i+1$ at time $j$. Temperature value of point $i$ at time $j+1, T_{i, j+1}$ is calculated using the temperature values at time $j$ using the temperature values of two neighboring points. We develop a formulation based on the explicit method to find the temperature distribution along the FGM layer whose thermal properties vary with the $x$ - 
coordinate. In order to discretize the time dependent heat conduction equation in space and time domains, the following central difference formulae are utilized.

$$
\begin{aligned}
\frac{\partial T}{\partial t} & =\frac{T_{i, j+1}-T_{i, j}}{\Delta t}+O(\Delta t), \\
\frac{\partial T}{\partial x} & =\frac{T_{i+1, j}-T_{i-1, j}}{2 \Delta x}+O\left(\Delta x^{2}\right), \\
\frac{\partial^{2} T}{\partial x^{2}} & =\frac{T_{i-1, j}-2 T_{i, j}+T_{i+1, j}}{(\Delta x)^{2}}+O\left(\Delta x^{2}\right) .
\end{aligned}
$$

When Eqs. (8-10) are substituted into Eq. (5) and after performing necessary mathematical manipulations, temperature at space $i$ and time $j+1, T_{i, j+1}$ is calculated through the following equation.

$$
\begin{aligned}
T_{i, j+1}= & T_{i, j}+\frac{\gamma_{1} \alpha_{0}}{2} \exp \left\{\left(\gamma_{1}-\gamma_{2}-\gamma_{3}\right) x_{i}\right\}\left(\frac{\Delta t}{\Delta x}\right)\left[T_{i+1, j}-T_{i-1, j}\right]+ \\
& +\alpha_{0} \exp \left\{\left(\gamma_{1}-\gamma_{2}-\gamma_{3}\right) x_{i}\right\}\left(\frac{\Delta t}{\Delta x^{2}}\right)\left[T_{i-1, j}-2 T_{i, j}+T_{i+1, j}\right] .
\end{aligned}
$$

Since explicit scheme calculates temperature distribution at time $j+1$ using the temperature information at time $j$, it is open to any instability problems. Since material properties of FGM layer vary with respect to spatial coordinate, the dimensionless mesh Fourier number for this material is not constant, so we need to define mesh Fourier number for all the grid points as,

$$
\tau_{i}=\frac{\alpha_{i} \Delta t}{(\Delta x)^{2}}
$$

where

$$
\alpha_{i}=\frac{k_{i}}{\rho_{i} c_{p i}}
$$

In Eq. (13), the subscript $i$ shows the properties of the FGM material at the spatial coordinate $x_{i}$. In order to obtain stable numerical solutions using explicit scheme, the following condition must be satisfied for all the grid points throughout the functionally graded layer. If this condition is not satisfied at least one of the grid points, the numerical solution of the overall system will not be stable and properly found.

$$
\tau_{i}=\frac{\alpha_{i} \Delta t}{(\Delta x)^{2}} \leq \frac{1}{2} .
$$




\subsection{Implicit method formulation}

As an implicit method, the Crank-Nicolson (C-N) scheme is considered for the transient thermal analysis of FGM layer. The Crank-Nicolson $(\mathrm{C}-\mathrm{N})$ scheme finds the solution of nodes $i-1, i$ and $i+1$ at time $j+1$ using the information available at nodes $i-1, i$ and $i+1$ at time $j$. In this method, temperature values of points $i-1, i, i+1$ at time $j+1$ are calculated utilizing the temperature values of points $i-1, i, i+1$ at time $j$. In this section, the formulation based on the implicit $(\mathrm{C}-\mathrm{N})$ method is provided for the thermal analysis of FGM layer. In order to discretize the time dependent heat conduction equation in space and time variables, the following central difference formulae are employed.

$$
\begin{aligned}
\frac{\partial T}{\partial t} & =\frac{T_{i, j+1}-T_{i, j}}{\Delta t}+O(\Delta t), \\
\frac{\partial T}{\partial x} & =\frac{T_{i+1, j}-T_{i-1, j}}{2 \Delta x}+O\left(\Delta x^{2}\right), \\
\frac{\partial^{2} T}{\partial x^{2}} & =\frac{T_{i-1, j}-2 T_{i, j}+T_{i+1, j}}{(\Delta x)^{2}}+O\left(\Delta x^{2}\right) .
\end{aligned}
$$

Utilizing the finite difference formula, the heat conduction equation is discretized as follows:

$$
\begin{aligned}
& \frac{T_{i, j+1}-T_{i, j}}{\Delta t}=\gamma_{1} \alpha_{0} \exp \left(\left(\gamma_{1}-\gamma_{2}-\gamma_{3}\right) x_{i}\right)\left(\frac{T_{i+1, j}-T_{i, j}}{\Delta x}\right)+ \\
& \quad+\frac{\alpha_{0} \exp \left(\left(\gamma_{1}-\gamma_{2}-\gamma_{3}\right) x_{i}\right)}{2(\Delta x)^{2}}\left(\left(T_{i+1, j+1}-2 T_{i, j+1}+T_{i-1, j+1}\right)+\left(T_{i+1, j}-2 T_{i, j}+T_{i-1, j}\right)\right)
\end{aligned}
$$

Rearrangement of Eq. (18) produces the following equation:

$$
-f_{i-1} T_{i-1, j+1}+g_{i} T_{i, j+1}-f_{i+1} T_{i+1, j+1}=f_{i-1} T_{i-1, j}+l_{i} T_{i, j}-S_{i+1} T_{i+1, j}
$$

where coefficients depending on space variables can be denoted by,

$$
\begin{gathered}
f_{i}=\frac{\alpha_{0}}{2(\Delta x)^{2}} \exp \left(\left(\gamma_{1}-\gamma_{2}-\gamma_{3}\right) x_{i}\right), \\
d_{i}=\frac{\gamma_{1}}{\Delta x} \alpha_{0} \exp \left(\left(\gamma_{1}-\gamma_{2}-\gamma_{3}\right) x_{i}\right), \\
g_{i}=\frac{1}{\Delta t}+2 f_{i}, \\
l_{i}=\frac{1}{\Delta t}-2 f_{i}-d_{i}, \\
s_{i}=d_{i}+f_{i} .
\end{gathered}
$$


Since the layer is made of functionally graded material, parameters $f_{i}, d_{i}, g_{i}, l_{i}$ and $s_{i}$ are not constant parameters. Eq. (19) is expanded in space and time grids using index parameters $j=1,2, \ldots, M$ and $i=1,2, \ldots, N$ where $M$ and $N$ indicates the boundary points of time and space grids. Hence, $T_{N M}$ shows the temperature of point $N$ at time $M$. In our parametric analyses, the final time of simulation is specified as $t_{f}=5 \mathrm{~s}$ and $0.01 \mathrm{~s}$ time intervals are used. The distance between two neighboring nodes is specified as $\Delta x=0.0005 \mathrm{~m}$. When Eq. (19) is expanded for $j=\{1,2,3, \ldots, M\}$ and $i=\{2,3,4, \ldots, N\}$, the equation set is obtained as follows:

$$
\left[\begin{array}{cccccc}
g_{2} & -f_{3} & 0 & 0 & \ldots & 0 \\
-f_{2} & g_{3} & -f_{4} & 0 & \ldots & 0 \\
0 & -f_{3} & g_{4} & -f_{5} & \ldots & 0 \\
0 & 0 & -f_{4} & g_{5} & -f_{6} & 0 \\
\ldots & \ldots & \ldots & -f_{N-3} & g_{N-2} & -f_{N-1} \\
0 & 0 & 0 & 0 & -f_{N-2} & g_{N-1}
\end{array}\right]\left\{\begin{array}{c}
T_{2, j} \\
T_{3, j} \\
T_{4, j} \\
T_{5, j} \\
\cdots \\
T_{N-1, j}
\end{array}\right\}=\left(\begin{array}{c}
\overline{z_{1}} \\
z_{2} \\
z_{3} \\
z_{4} \\
\ldots \\
\overline{z_{N-2}}
\end{array}\right) .
$$

where

$$
\begin{gathered}
\overline{z_{1}}=f_{1} T_{1, j}+l_{2} T_{2, j}+s_{3} T_{3, j}+f_{1} T_{1, j+1}, \\
z_{2}=f_{2} T_{2, j}+l_{3} T_{3, j}+s_{4} T_{4, j}, \\
z_{3}=f_{3} T_{3, j}+l_{4} T_{4, j}+s_{5} T_{5, j}, \\
\overline{z_{N-2}}=f_{N-2} T_{N-2, j}+l_{N-1} T_{N-1, j}+s_{N} T_{N, j}+f_{N} T_{N, j+1} .
\end{gathered}
$$

The algebraic equation system given by Eq. (25) is solved for all time steps $j=1,2, \ldots, M$. The parameters in coefficient matrix are not constants since they depend on the material properties in FGM layer. In Eqs (26) - (29), $T_{1, j+1}$ and $T_{N, j+1}$ are known temperature values from the specified boundary conditions. The algebraic equation system is solved for unknown temperatures at interior points.

\subsection{Finite element method (FEM)}

The parametric finite element analyses are performed with ANSYS Parametric Design Language (APDL) [29]. 8-node PLANE77 element is utilized in simulations. This element is a higher order version of the 2-D, 4-node thermal element PLANE55 [29]. The element has one degree of freedom which is temperature at each node. The 8-node thermal element is appropriate to a 2-D, steady-state or transient thermal analyses. The geometry, node locations and the coordinate system for PLANE77 is depicted in Fig 3(a). A triangular shape option may be formed by merging three nodes at the same point. Modelling functionally graded layer using finite elements requires assigning continuously varying material properties into the layer. The most conventional way to model graded material inhomogeneity involves the use of conventional homogenous elements in successive layers of the mesh, containing own material properties [30]. Hence, stepwise change in properties along the direction of gradation is satisfied. Such ways have already been used for many researchers [31-32], hence we have 
adopted this model in the present study. Constructed finite element mesh for transient heat conduction analysis of the FGM layer is depicted in Fig. 3(b).
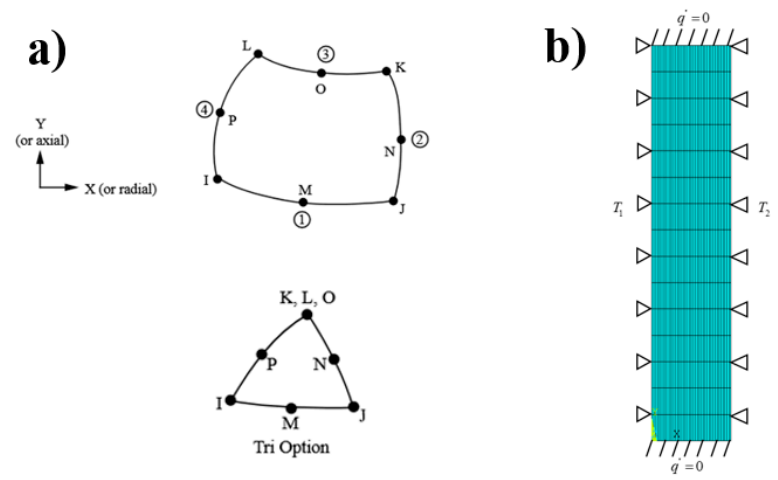

Fig. 3. (a) Two-dimensional 8-node thermal solid element available in ANSYS [29] (b) Constructed finite element mesh for the FGM layer

\section{Results}

In this section, we provide temperature distribution results. Firstly, obtained results based on three different methods are compared with each other to verify the developed numerical formulations. Two different boundary and initial conditions are applied to the layer and they are labeled as Case 1 and Case 2 as mentioned in section 2. In Case 1, the temperature at left and right surfaces of the layer is kept constant as $0 \mathrm{~K}$ and FGM layer is loaded by a sinusoidal temperature profile initially. In Case 2, the temperature at left surface of FGM layer is kept constant as $2 T_{0} \mathrm{~K}$ whereas the temperature at right surface is kept constant as $T_{0} \mathrm{~K}$. In both cases, the influences of inhomogeneity constants for thermal conductivity, mass density and thermal capacitance on transient temperature distribution in an FGM layer are examined. Before analyzing the effect of inhomogeneity constants, the temperature distribution in the FGM layer is analyzed and compared with that obtained in a homogeneous layer which is composed of $100 \%$ Ti-6Al-4V. Fig. 7(a) and 7(b) respectively show the contours of temperature distribution in homogenous layer and FGM layer in Case 1 condition with respect to time. Temperature values in the layer is normalized utilizing $T_{0}=1000 \mathrm{~K}$. Hence, $T(x) / T_{0}$ results are presented in parametric studies. The coordinate parameter along the layer is normalized by the following equation,

$$
\bar{x}=\frac{2 x}{L}-1
$$

According to these figures, the homogenous layer is cooling faster than the FGM layer. Moreover, cooling in homogenous layer appears symmetric whereas cooling in FGM layer appears not symmetric as predicted. Since right surface of the layer composed of $\mathrm{ZrO}_{2}$, conduction towards right surface gradually decreases. The low thermal conductivity of $\mathrm{ZrO}_{2}$ plays an important role on observing such a behavior. Fig. 7(c) and (d) show the contours of temperature distribution of homogenous layer and FGM layer, respectively when Case 2 condition is applied to the layer. When Figs 7(c) and (d) are examined, temperature at the right surface of homogenous layer diffuses faster into the thickness of the layer when compared to that of FGM layer. Therefore, the cool region emerging around the metallic surface of FGM layer is larger than that of homogenous layer. 
Fig.8 (a) and (b) show the temperature distribution in a homogenous layer loaded by Case 1 and Case 2 conditions with respect to time for the discussed numerical methods. It can be inferred from figures that results of developed code for explicit and implicit schemes are in a very good agreement with those of finite element analysis. In addition, Fig.9 (a) and (b) illustrate the temperature distribution in a functionally graded layer loaded by Case 1 and Case 2 conditions with respect to time. Since layer is made of FGM, cooling curves observed from Fig. 9(a) is not symmetric, and heating curves appearing in Fig. 9(b) is different from that appearing in Fig. 8(b). It can be inferred from Fig. 9(a) and (b) that obtained results for the FGM layer are in a very good agreement with the results of finite element method similar to the homogeneous case.

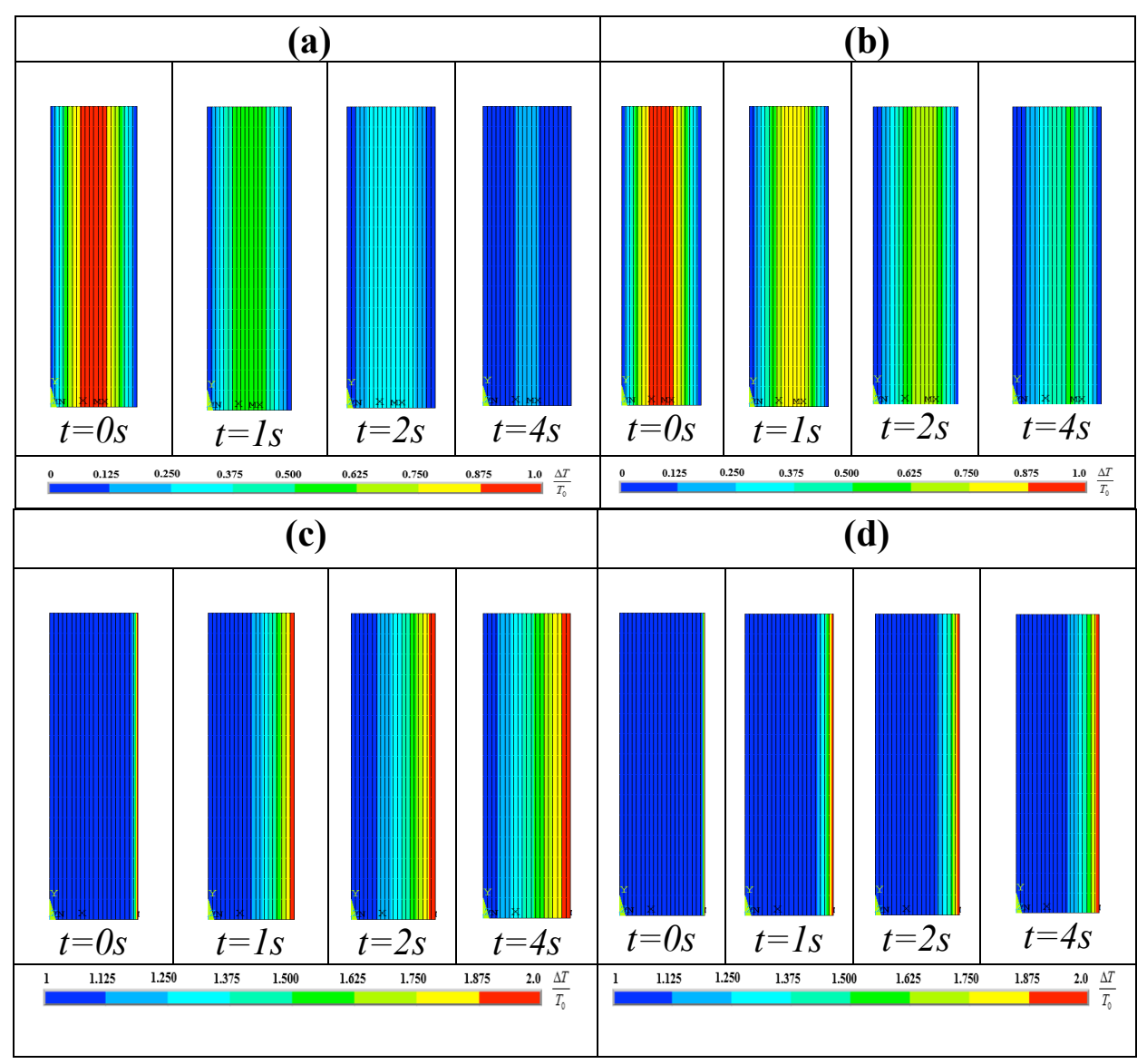

Fig. 7. (a) Temperature contours in homogenous layer in Case 1, (b) Temperature contours in FGM layer in Case 1, (c) Temperature contours in homogenous layer in Case 2, (d) Temperature contours in FGM layer in Case 2. 

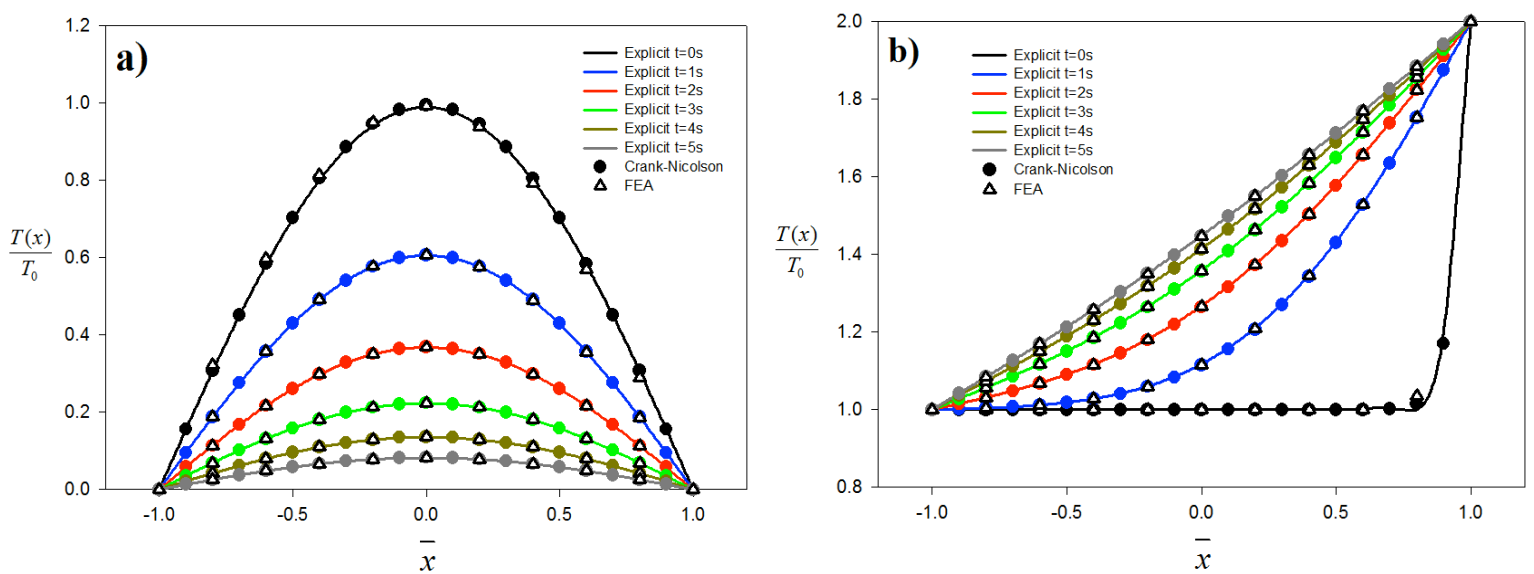

Fig. 8. Temperature distribution in homogenous layer made of Ti-6Al-4V using different numerical techniques with respect to time $\gamma_{1}=0, \gamma_{2}=0, \gamma_{3}=0$.
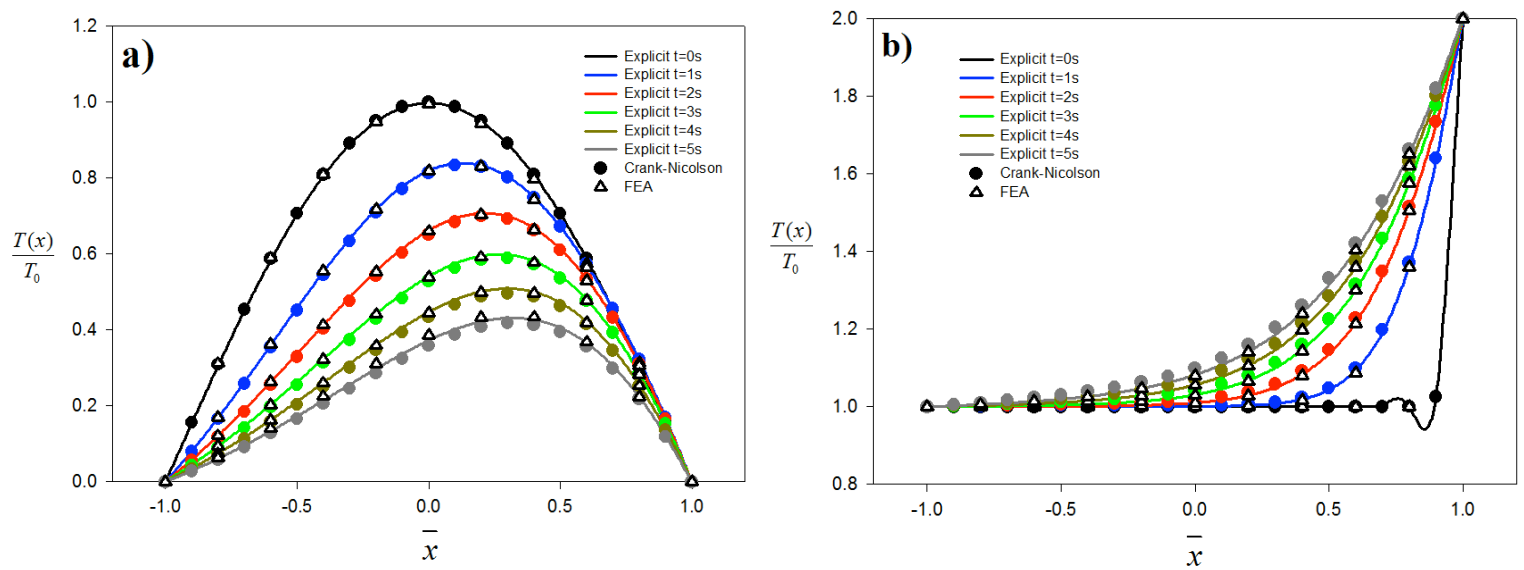

Fig. 9. Temperature distribution in FGM layer made of Ti-6Al-4V and $\mathrm{ZrO}_{2}$ using different numerical techniques with respect to time $\gamma_{1}=-2.185, \gamma_{2}=-0.2724, \gamma_{3}=0.2366$.

The influence of each inhomogeneity constants on the temperature distribution is evaluated and compared with each other. This is performed by analyzing the temperature at the midpoint of the layer $\left(T_{M P}=T(x / 2)\right)$ while varying each inhomogeneity parameter, individually. Fig. 16 shows the variation of normalized temperature with respect to time for the midpoint of the layer. Shown in Figs. 10 (a)-(d), the midpoint temperature is decreasing with time under Case 1 condition whereas it is increasing under Case 2 condition. For both cases, the variation $\gamma_{2} L$ and $\gamma_{3} L$ have similar influences and increase in those parameters results in higher midpoint temperature while cooling condition in Case 1 and lower midpoint temperature while heating condition in Case 2. Furthermore, the opposite is true for the increase in $\gamma_{1} L$. Decrease in $\gamma_{2} L$ and $\gamma_{3} L$ leads to lower midpoint temperature in Case 1 in which cooling occurs and much higher midpoint temperature in Case 2 where heating happens. On the contrary, decrease in $\gamma_{1} L$ causes higher midpoint temperature in Case 1 and lower temperature in Case 2. The main reason behind this behavior is that the inhomogeneity constant solely participate in transient heat conduction Eq. (5) ahead of $\frac{\partial T}{\partial x}$ term and $\gamma_{1}$ exponentially contributes to the thermal diffusivity (see Eq. (6)) in a positive way, however 
the opposite is true for $\gamma_{2}$ and $\gamma_{3}$. Following figure (see Fig. 11) illustrates the midpoint temperature when inhomogeneity constants $\gamma_{1} L, \gamma_{2} L$ and $\gamma_{3} L$ are varied together. Hence, combined effect of inhomogeneity parameters on midpoint temperature value can be clearly observed. When $\gamma_{1} L, \gamma_{2} L$ and $\gamma_{3} L$ are increased together, the midpoint temperature in Case 1 rises and Case 2 reduces. Similarly, decrease in $\gamma_{1} L, \gamma_{2} L$ and $\gamma_{3} L$ together leads to lower midpoint temperature value in Case 1 and higher midpoint temperature value in Case 2 . We can draw such a conclusion from the findings that the alteration of $\gamma_{2} L$ and $\gamma_{3} L$ are more dominant on midpoint temperature value in layer than that of $\gamma_{1} L$.
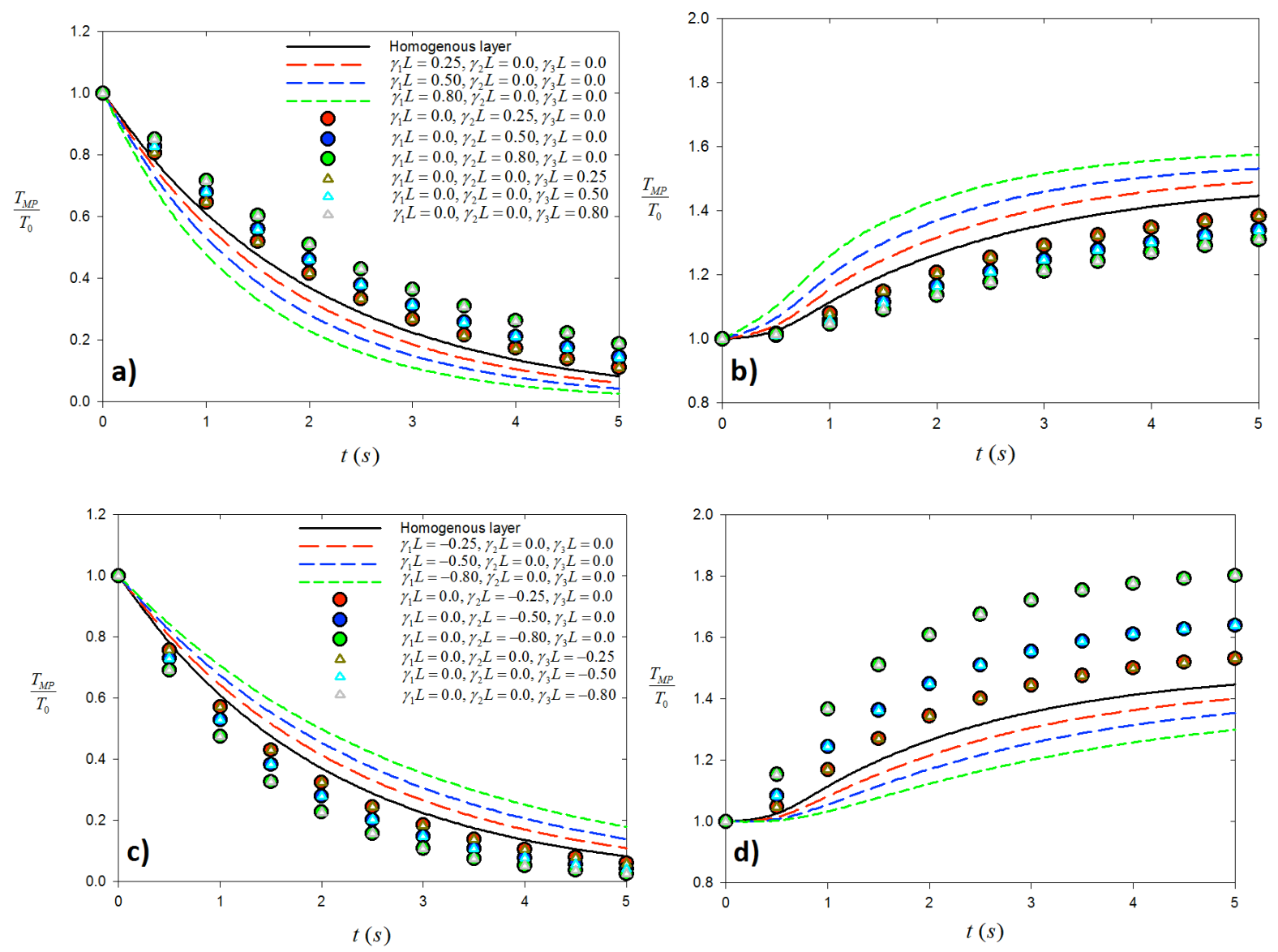

Fig. 10. The midpoint temperature value in FGM layer with respect to time (a) for increasing values of inhomogeneity constants $\gamma_{1} L, \gamma_{2} L$ and $\gamma_{3} L$ individually (a) Case 1 (b) Case 2; (c) for decreasing values of inhomogeneity constants $\gamma_{1} L, \gamma_{2} L$ and $\gamma_{3} L$ individually (c) Case 1

(d) Case 2. 

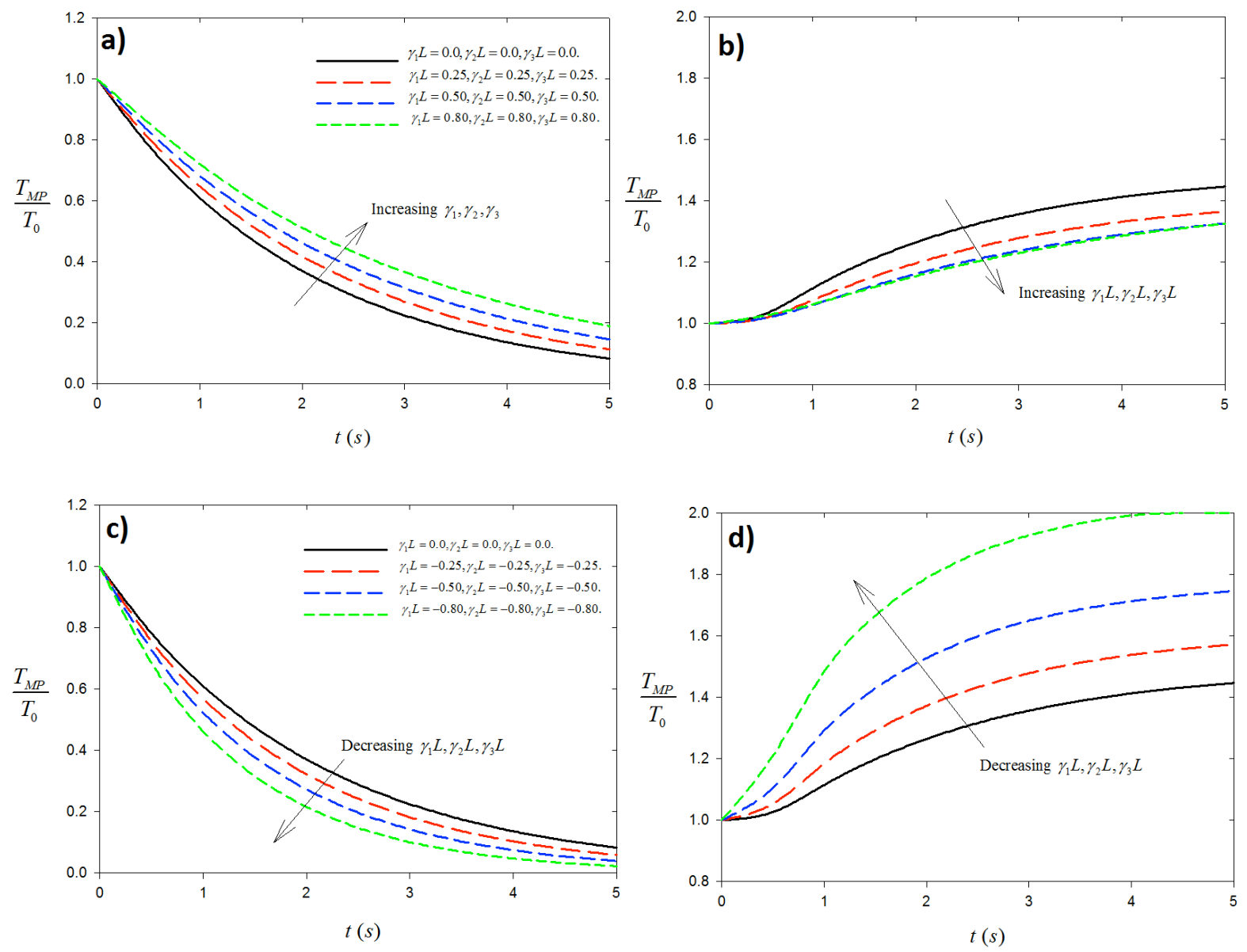

Fig. 11. The midpoint temperature value in FGM layer with respect to time (a) for increasing values of $\gamma_{1} L, \gamma_{2} L$ and $\gamma_{3} L$ together in Case 1 (b) for increasing values of $\gamma_{1} L, \gamma_{2} L$ and $\gamma_{3} L$ together in Case 2 (c) for decreasing values of $\gamma_{1} L, \gamma_{2} L$ and $\gamma_{3} L$ together in Case 1 (d) for decreasing values of $\gamma_{1} L, \gamma_{2} L$ and $\gamma_{3} L$ together in Case 2.

In order to see which of the inhomogeneity parameter is the most dominant, the normalized values of midpoint temperature in the layer at $t=5 \mathrm{~s}$ are provided in Table 3. Midpoint temperatures regarding individual and combined alteration of the inhomogeneity constants are given in a tabular format. Percent difference is shown by $\varepsilon \%$ and it is calculated with respect to the temperature in which combined inhomogeneity take place $\left(\gamma_{1} L=\gamma_{2} L=\gamma_{3} L=0.8\right.$ and $\left.\gamma_{1} L=\gamma_{2} L=\gamma_{3} L=-0.8\right)$. We have denoted this temperature using (*) as the referencing state. In Case 1 and 2, when dimensionless temperature values are compared, increase in $\gamma_{2} L$ and $\gamma_{3} L$ have equal influence on temperature and they lead to get closer temperatures to the temperature at referencing state. For both cases, decreases in $\gamma_{2} L$ and $\gamma_{3} L$ have the same effect and they are more influential than the change in $\gamma_{1} L$. It can be quantitatively inferred from Table 3 that the inhomogeneity constants $\gamma_{2} L$ and $\gamma_{3} L$ have greater influence on midpoint temperature than that of $\gamma_{1} L$ and they equally influence the temperature value. 
Table 3. Normalized midpoint temperature values at $t=5 \mathrm{~s}$.

\begin{tabular}{|l|c|c|c|c|}
\hline & $\begin{array}{c}\text { Case 1 } \\
T_{M P} / T_{0}(t=5 s)\end{array}$ & $\varepsilon \%$ & $\begin{array}{c}\text { Case 2 } \\
T_{M P} / T_{0}(t=5 s)\end{array}$ & $\varepsilon \%$ \\
\hline Homogenous Layer & 0.08291 & 56.23 & 1.44732 & 9.13 \\
\hline$\gamma_{1} L=0.8, \gamma_{2} L=0.0, \gamma_{3} L=0.0$. & 0.02573 & 86.41 & 1.57624 & 18.85 \\
\hline$\gamma_{1} L=0.0, \gamma_{2} L=0.8, \gamma_{3} L=0.0$. & 0.18846 & 0.517 & 1.30999 & 1.22 \\
\hline$\gamma_{1} L=0.0, \gamma_{2} L=0.0, \gamma_{3} L=0.8$. & 0.18846 & 0.517 & 1.30999 & 1.22 \\
\hline$\gamma_{1} L=0.8, \gamma_{2} L=0.8, \gamma_{3} L=0.8$. & $\mathbf{0 . 1 8 9 4 4 ( * )}$ & - & $\mathbf{1 . 3 2 6 2 3 ( * )}$ & - \\
\hline$\gamma_{1} L=-0.8, \gamma_{2} L=0.0, \gamma_{3} L=0.0$. & 0.17870 & 712.02 & 1.29911 & 35.05 \\
\hline$\gamma_{1} L=0.0, \gamma_{2} L=-0.8, \gamma_{3} L=0.0$. & 0.02523 & 14.06 & 1.80274 & 9.86 \\
\hline$\gamma_{1} L=0.0, \gamma_{2} L=0.0, \gamma_{3} L=-0.8$. & 0.02523 & 14.06 & 1.80274 & 9.86 \\
\hline$\gamma_{1} L=-0.8, \gamma_{2} L=-0.8, \gamma_{3} L=-0.8$. & $\mathbf{0 . 0 2 2 1 2 ( * )}$ & - & $\mathbf{2 . 0 0 0 0 0 0}(*)$ & - \\
\hline
\end{tabular}

\section{Conclusions}

In this study, the transient heat conduction in a functionally graded layer is examined using various computational techniques. Heat conduction equation for the functionally graded layer is derived without any simplification for the inhomogeneity. Hence, inhomogeneity parameters controlling thermal conductivity, specific heat capacitance and mass density involve. Time dependent temperature distribution in the functionally graded layer is obtained by applying explicit and implicit schemes to the time dependent variable coefficient partial differential equation (PDE). Developed formulations for the functionally graded layer are implemented via computer codes in MATLAB. In addition, functionally graded layer is modeled in ANSYS [29] and transient heat conduction analysis is carried out. Two different cases of boundary and initial conditions are considered. In the first case, the layer is loaded by the sinusoidal temperature initially while temperatures at surfaces of the layer are kept constant, so cooling behavior is observed. In the second case, one of the surfaces of the layer is subjected to $2 T_{0} \mathrm{~K}$ while other surface is hold at $T_{0} \mathrm{~K}$, hence heating behavior is seen. In both cases, the results of developed formulations are compared with those obtained by finite element analysis and a very good agreement is accomplished. Then, a series of parametric analyses are conducted to investigate the separate effects of inhomogeneity constants on temperature distributions. The results of this study can guide the material specialists to determine the materials to be utilized in an FGM layer in thermal barriers according to the desired temperature distribution. Some concluding remarks are summarized as follows:

- Decrease in $\gamma_{1} L$ leads to higher temperatures towards the ceramic side in an FGM layer.

- $\quad$ The change in $\gamma_{2} L$ and $\gamma_{3} L$ affects the temperature in an equal manner. Increase in $\gamma_{2} L$ and $\gamma_{3} L$ leads to slow temperature change whereas decrease in those parameters causes a fast change. 
- The order of the dominance of the inhomogeneity constants on thermal conductance analysis is summarized as: $\gamma_{2} L=\gamma_{3} L>\gamma_{1} L$.

\section{References}

[1] Miyamoto, Y., Kaysser, W.A., Rabin, B.H., Kawasaki, A., Ford, R.G., Functionally Graded Materials Design, Processing and Applications. Springer Science \& Business Media, New York 1999.

[2] Naebe, M., Shirvanimoghaddam, K., Functionally graded materials: A review of fabrication and properties. Applied Materials Today, 5, 223-245, 2016.

[3] Bellur-Ramaswamy, R.S., Haber, R., Sobh, N.A., Tortorelli, D.A., Modelling and process optimization for functionally graded materials. International Journal for Numerical Methods in Engineering, 62, 186-204, 2005.

[4] Fukui, Y., Takashima, K., Ponton, C.B., Measurement of Young's modulus and internal

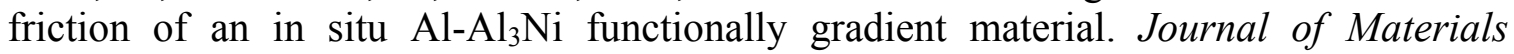
Science, 29(9), 2281-2288, 1994.

[5] Abbas, M.R., Uday, M.B., Noor, A.M., Ahmad, N., Rajoo, S., Microstructural evaluation of a slurry based Ni/YSZ thermal barrier coating for automotive turbocharger turbine application. Materals \& Design, 109, 47-56, 2016.

[6] Dhineshkumar, S.R., Duraiselvam, M., Natarajan, S., Panwar, S.S., Jena, T., Khan, M.A., Enhancement of strain tolerance of functionally graded $\mathrm{LaTi}_{2} \mathrm{Al}_{9} \mathrm{O}_{19}$ thermal barrier coating through ultra-short pulsed based laser texturing. Surface \& Coatings Technology, 304, 263-271, 2016.

[7] Naga, S.M., Awaad, M., El-Maghraby, H.F., Hassan, A.M., Elhoriny, M., Killinger, A., Gadow, R., Effect of $\mathrm{La}_{2} \mathrm{Zr}_{2} \mathrm{O}_{7}$ coat on the hot corrosion of multi-layer thermal barrier coatings. Materials \& Design, 102, 1-7, 2016.

[8] Daikh, A.A., Megueni, A., Thermal behavior of Functionally Graded Materials. In: $5^{\text {th }}$ International Conference on Welding Non Destructive Testing and Materials and Alloys Industry, Oran, Algeria, 2016.

[9] Kuroda, Y., Kusaka, K., Moro, A., Togawa, M., Evaluation tests of $\mathrm{ZrO}_{2} / \mathrm{Ni}$ functionally gradient materials for regeneratively cooled thrust engine applications. Holt J.B., Koizumi M., Hirai T., Munir Z.A. (Eds.), Ceramic Transactions, 34, Functionally Gradient Materials, American Ceramic Society, Westerville, Ohio, 289-296, 1993.

[10] Reddy, J.N., Chin, C.D., Thermomechanical analysis of functionally graded cylinders and plates. Journal of Thermal Stresses, 21(6), 593-626, 1998.

[11] Yang, Y., Temperature Dependent Thermoelastic Analysis of Multi-dimensional Functionally Graded Materials, Ph.D. Dissertation, University of Pittsburgh, USA, 2015.

[12] Hosseini, S.M., Akhlaghi, M., Shakeri, M., Transient heat conduction in a functionally graded thick hollow cylinders by analytical method. Heat and Mass Transfer, 43, 669675, 2007. 
[13] Bahtui, A., Eslami, M.R., Generalized coupled thermoelasticity of functionally graded cylindrical shells. International Journal for Numerical Methods in Engineering, 69, 676697, 2007.

[14] Zhao, X., Liew, K.M., An element-free analysis of mechanical and thermal buckling of functionally graded conical shell panels. International Journal for Numerical Methods in Engineering, 86, 269-285, 2011.

[15] Sharma, R., Jadon, V.K., Singh, B., Analysis of temperature field in a composite functionally graded material plate by finite element method. International Journal of Advances in Materials Science and Engineering (IJAMSE), 4(4), 41-47, 2015.

[16] Cho, J.R., Oden, J.T., Functionally graded material: a parametric study on thermal-stress characteristics using the Crank-Nicolson-Galerkin scheme. Computer Methods in Applied Mechanics and Engineering, 188, 17-38, 2000.

[17] Nemat-Alla, M., Reduction of thermal stresses by composition optimization of twodimensional functionally graded materials. Acta Mechanica, 208, 147-161, 2009.

[18] Sladdek, J., Sladdek, V., Zhang, C., Transient heat conduction analysis in a functionally graded materials by the meshless local boundary integral equation method. Computational Materials Science, 28, 494-504, 2003.

[19] Sadowski, T., Nakonieczny, K., Thermal shock response of FGM cylindrical plates with various grading patterns. Computational Materials Science, 43: 171-178, 2008.

[20] Nakonieczny, K., Sadowski, T., Modelling of 'thermal shocks' in composite materials using meshfree FEM. Computational Materials Science, 44, 1307-1311, 2009.

[21] Li, G., Guo, S., Zhang, J., Li, Y., Han, L., Transient heat conduction analysis of functionally graded materials by a multiple reciprocity boundary face method. Engineering Analysis with Boundary Elements, 60, 81-88, 2015.

[22] Li, M., Wen, P.H., Finite block method for transient heat conduction analysis in functionally graded media. International Journal for Numerical Methods in Engineering, 99, 372-390, 2014.

[23] Olatunji-Ojo, A.O., Boetcher, S., Cundari, T.R., Thermal conduction analysis of layered functionally graded materials. Computational Materials Science, 54, 329-335, 2012.

[24] Jin, Z., Heat Conduction in a Functionally Graded Plate Subjected to Finite Cooling/Heating Rates: An Asymptotic Solution. Materials, 4(12), 2108-2118, 2011.

[25] Chan, Y-S., Paulino, G.H., Fannjiang, A.C., Gradient Elasticity Theory for Mode III Fracture in Functionally Graded Materials - Part II: Crack Parallel to the Material Gradation. ASME Journal of Applied Mechanics, 75(0611015), 1-11, 2008.

[26] Balci, M.N., Dag, S., Yildirim, B., Subsurface stresses in graded coatings subjected to frictional contact with heat generation. Journal of Thermal Stresses, 40(4), 517-534, 2017.

[27] Chen, P., Chen, S., Thermo-mechanical contact behavior of a finite graded layer under a sliding punch with heat generation. International Journal of Solids and Structures, 50, 1108-1119, 2013.

[28] Fujimoto, T., Noda, N., Influence of the compositional profile of functionally graded material on the crack path under thermal shock. Journal of the American Ceramic Society, 84(7), 1480-1486, 2001. 
[29] ANSYS, ANSYS Basic Analysis Procedures Guide, release 15.1, ANSYS Inc., Canonsburg, PA, USA, 2015.

[30] Burlayenko, V.N., Altenbach, H., Sadowski, T., Dimitrova, S.D., Bhaskar, A., Modelling functionally graded materials in heat transfer and thermal stress analysis by means of graded finite elements. Applied Mathematical Modelling, 45,422-438, 2017.

[31] Fuchiyama, T., Noda, N. Analysis of thermal stresses in a plate of functionally gradient material. JSAE Review, 16, 373-387, 1995.

[32] Anlas, G., Santare, N.H., Lambros, J., Numerical calculation of stress intensity factors in a functionally graded materials. International Journal of Fracture, 104, 131-143, 2000. 